\title{
Prenatal arachidonic acid exposure and selected immune-related variables in childhood
}

\author{
Chantal E. H. Dirix ${ }^{1}$, Janneke G. F. Hogervorst ${ }^{2}$, Patrick Rump ${ }^{1,3}$, Johannes J. E. Hendriks ${ }^{4}$, Maaike Bruins ${ }^{5}$ \\ and Gerard Hornstra 6 *, \\ ${ }^{1}$ Department of Human Biology, Faculty of Health, Medicine and Life Sciences, Maastricht University, Maastricht, \\ The Netherlands \\ ${ }^{2}$ Department of Epidemiology, Faculty of Health, Medicine and Life Sciences, Maastricht University, Maastricht, The Netherlands \\ ${ }^{3}$ Department of Genetics, University Medical Center Groningen, University of Groningen, Groningen, The Netherlands \\ ${ }^{4}$ Department of Paediatrics, University Hospital Maastricht, Maastricht, The Netherlands \\ ${ }^{5}$ DSM Food Specialties, R\&D-FTD, Delft, The Netherlands \\ ${ }^{6}$ Nutrition and Toxicology Research Institute Maastricht, Faculty of Health, Medicine and Life Sciences, Maastricht University, \\ Maastricht, The Netherlands \\ ${ }^{7}$ NUTRI-SEARCH, Gronsveld, The Netherlands
}

(Received 16 April 2008 - Revised 13 October 2008 - Accepted 15 October 2008 - First published online 28 January 2009)

Arachidonic acid (AA) is considered essential in fetal development and some of its metabolites are thought to be important mediators of the immune responses. Therefore, we studied whether prenatal exposure to AA is associated with some immune-related clinical conditions and plasma markers in childhood. In 280 children aged 7 years, atopy, lung function and plasma inflammation markers were measured and their relationships with early AA exposure were studied by linear and logistic regression analyses. AA exposure was deduced from AA concentrations in plasma phospholipids of the mothers collected at several time points during pregnancy and at delivery, and in umbilical cord plasma and arterial and venous wall phospholipids. In unadjusted regression analyses, significant positive associations were observed between maternal AA concentrations at 16 and 32 weeks of pregnancy (proxies for fetal AA exposure) and peak expiratory flow decline after maximal physical exercise and plasma fibrinogen concentrations of their children, respectively. However, after correction for relevant covariables, only trends remained. A significant negative relationship was observed between AA concentrations in cord plasma (reflecting prenatal AA exposure) and the average daily amplitude of peak expiratory flow at rest, which lost significance after appropriate adjustment. Because of these few, weak and inconsistent relationships, a major impact of early-life exposure to AA on atopy, lung function and selected plasma inflammation markers of children at 7 years of age seems unlikely.

Prenatal arachidonic acid: Atopy: Lung function: Plasma inflammation markers

There is increasing evidence that exposure to nutritional factors, such as n-6 and n-3 PUFA, during the perinatal period may influence the development of the immune system and subsequent immune competence ${ }^{(1)}$. The $n-6$ and $n$-3 PUFA are important structural components of cell membranes and particularly arachidonic acid (AA) is prominently present in immune cell membrane phospholipids $(\mathrm{PL})^{(2,3)}$. AA may influence the developing immune system because it is the precursor of PGE2, which is thought to be an important mediator of immune responses ${ }^{(4,5)}$. During pregnancy, the maternal essential fatty acid (EFA) status declines and because the EFA status of the neonate is strongly correlated with the EFA status of its mother ${ }^{(6)}$, the AA supply to the developing fetus and its immune system may not always be optimal.
Previous studies investigated the relationship between the maternal intake of long-chain $n$-3 PUFA during pregnancy and immune-related variables of their offspring ${ }^{(7,8)}$. However, only a few studies considered the relationship between the prenatal long-chain $n-6$ PUFA status, especially AA, and immune-related variables, such as atopy later in life. Galli et al. observed that babies who developed atopic disease after 1 year of birth had 20-40\% lower levels of AA in their serum cord blood cells in comparison with non-atopic babies $^{(9)}$. In contrast, Yu et al. found no significant differences in any of the fatty acid levels, measured in umbilical venous blood samples, of babies who did or did not develop allergic diseases during the first 6 years of life ${ }^{(10)}$. Furthermore, Newson et al. studied the relationship between levels of various $n-3$ and $n-6$ series fatty acids, including AA, in

Abbreviations: AA, arachidonic acid; CRP, C-reactive protein; EFA, essential fatty acid; PEF, peak expiratory flow; PL, phospholipid; SES, socio-economic status. * Corresponding author: Dr Gerard Hornstra, fax +3143 3560535, email G.Hornstra@ Nutrisearch.NL 
erythrocyte PL of women in late pregnancy and in erythrocyte PL of the umbilical cord of their children, and the prevalence of wheezing and eczema of the child from birth until 6 months and from age 30-42 months ${ }^{(11)}$. No significant associations were observed after adjustment for covariables. Thus, the relationship between prenatal AA concentrations and later immune-related variables remains relatively unexplored. Therefore, we investigated in a mother-child cohort the associations between the AA levels of maternal plasma PL, during early, middle and late pregnancy (as proxies for fetal AA exposure), and several immune-related clinical conditions and inflammation markers of the child at 7 years of age. In addition, we also investigated if these immune-related variables were associated with PL AA concentrations collected in cord plasma and vessel walls of the newborns, all assumed to reflect prenatal AA exposure.

\section{Subject and methods}

\section{Study population and design}

The Maastricht Essential Fatty Acid Birth (MEFAB) cohort resulted from observational studies performed during 1990-7, which investigated the associations between maternal or neonatal EFA status during pregnancy and pregnancy outcome in approximately 1200 pregnant women and most of their infants $^{(12,13)}$. In 1997, the parents of the 750 singleton infants born between 1990 and 1994 were approached for an extensive follow-up and eventually 305 children participated in these studies between 1997 and $2000^{(14,15)}$. Results of this follow-up were also entered in the MEFAB database, which provided all data for the present study. Children with an unknown gestational age at birth or who were born before 37 weeks gestational age or after 43 weeks of pregnancy were excluded ( $n 25)$. Ultimately, the data of 280 children and their mothers were available for the present statistical analysis. Unadjusted and multivariableadjusted regression models were applied to explore if maternal or neonatal AA concentrations, measured during pregnancy and/or directly after delivery, are related to childhood immune-related variables.

\section{Blood and tissue sampling and fatty acid measurements}

Maternal venous blood samples were collected in EDTA tubes around the 16th, 22nd and 32nd week of pregnancy, and immediately after delivery. Umbilical cord blood and a piece of the umbilical cord were obtained immediately after parturition. About 7 years later, after an overnight fast, venous blood from the children was collected by venepuncture in EDTA-treated evacuated tubes. Plasma was separated from blood cells by centrifugation. Plasma and umbilical tissue samples were stored under $\mathrm{N}_{2}$ at $-80^{\circ} \mathrm{C}$ until analysis. The fatty acid composition of PL isolated from plasma and cord vein and artery walls was determined by capillary GLC as described elsewhere ${ }^{(6,16)}$. Fatty acids are expressed as relative values ( $\%$ by weight of total identified PL-associated fatty acids).

\section{Explanatory variables}

Four explanatory neonatal variables were taken to reflect prenatal AA exposure, i.e. the relative AA concentrations of
PL isolated from umbilical plasma and from the walls of the cord vein and artery, and the difference between these latter two concentrations, which we considered a proxy for fetal AA consumption ${ }^{(17)}$. The AA concentrations of maternal plasma PL at approximately 16, 22 and 32 weeks of gestation and at delivery were applied as four additional explanatory variables. These variables are taken to reflect the AA status of the fetus during gestation, since the maternal and neonatal EFA statuses are strongly correlated ${ }^{(6)}$.

\section{Dependent variables}

The following immune-related variables were modelled as dependent variables in the regression analyses: presence of atopic clinical conditions, peak expiratory flow (PEF) outcomes and plasma concentrations of a number of inflammation markers (all described later).

Atopy assessment. For the atopy assessment of the children, parents completed an atopy questionnaire, based on the Maastricht Atopy List ${ }^{(18)}$ and the well-validated ${ }^{(19,20)}$ International Study of Asthma and Allergies in Childhood (ISAAC) questionnaire ${ }^{(21)}$. This questionnaire included queries about typical atopy-related symptoms, categorised per atopic organ ${ }^{(22)}$, such as 'Has your child ever had allergic complaints such as eczema, hay fever, and/or food allergy?', 'Has your child ever had asthma or asthmatic bronchitis?' and 'Has your child ever had wheezing or whistling in the chest?'. In addition, the following question about allergy tests was included: 'Has your child ever undergone an allergy test like a skin-prick test, radioallergosorbent test (RAST), elimination test or otherwise? If yes, what was the test result?'. From these questionnaires, the presence of atopy was evaluated by a paediatric pulmonologist (J. J. E. H.) and scored 'yes' or 'no' when, respectively, more than one or none of the above-mentioned clinical conditions were conclusively manifest. Children whose diagnosis was inconclusive (scoring only one of the above-mentioned clinical conditions) were excluded from the analysis to prevent bias introduced by misclassification.

Peak flow measurements. Asthma is a chronic inflammatory disorder of the airways in which many different cell types play a role. In susceptible individuals this inflammation causes symptoms which are usually associated with widespread but variable airflow obstruction, that is often reversible either spontaneously or with treatment, and causes an associated increase in airway responsiveness to a variety of stimuli $^{(23)}$. PEF measurements are often used in everyday clinical and epidemiological environments for measuring the severity in airflow obstruction of asthma, to characterise the clinical trial population and to describe the response to treatment ${ }^{(24)}$. In addition, investigators of previous studies observed that in children with asthmatic symptoms atopy was associated with a greater within-day and between-day variation in $\mathrm{PEF}^{(25,26)}$. To assess pulmonary function in the present study, PEF was measured using a Mini-Wright peak flow meter. Measurements were carried out at rest (at home) and before and after maximal physical exercise (at the laboratory), both after instructions of the research team. Under the supervision of the parents, PEF measurements at rest were performed for 2 weeks, five times per d during the morning (between 07.00 and 09.00 hours) and five times per d during 
the evening (between 19.00 and 21.00 hours). Each day, the highest PEF value in the morning and the highest value in the evening were recorded. To increase the reliability of the measurements, these values were only accepted if at least two other PEF values (of that series of five measurements) fell within a range of $10 \%$ of that highest value. If this was not the case, this procedure was repeated with the next highest PEF value, etc, until two PEF values fell within the $10 \%$ range of that next highest $\mathrm{PEF}$ value, otherwise a missing value was generated. The average highest PEF value at rest in the morning (PEF morning) was calculated for the 2-week measurement period. In addition, the PEF daily amplitude was calculated as the difference between the highest morning and highest evening PEF value and expressed as a percentage of the mean ${ }^{(27)}$. The average daily PEF amplitude (PEF amplitude) was calculated over the 2-week measurement period. To measure PEF decline after exercise provocation (PEF exercise), PEF measurements were performed at the laboratory three times before, and three times at 2, 5, 10 and 15 min after reaching maximal exercise with the Bruce treadmill test ${ }^{28,29)}$, of which the highest PEF value was recorded each time. PEF decline after exercise provocation was calculated as: $\left(\left(\mathrm{PEF}_{\text {highest value before exercise }}-\mathrm{PEF}_{\text {lowest value after }}\right.\right.$ maximal exercise $) / \mathrm{PEF}_{\text {highest value before exercise }) \times 100 \%}{ }^{(30)}$.

Plasma inflammation markers. The following factors are involved in different processes of the inflammatory response and were therefore chosen as dependent variables: fibrinogen $(\mathrm{g} / \mathrm{l})^{(31)}$, C-reactive protein $(\mathrm{CRP} ; \mathrm{mg} / \mathrm{l})^{(32)}$, leptin $(\mu \mathrm{g} / \mathrm{l})^{(33)}$ and von Willebrand factor concentrations ${ }^{(34)}$. In addition, total leucocyte counts $\left(\times 10^{9} / 1\right.$ blood) were measured, as well as the absolute and relative amounts of lymphocytes, granulocytes and monocytes (\% of total leucocytes) ${ }^{(35)}$.

Fibrinogen was assayed by the Clauss method ${ }^{(36)}$ and CRP was measured with an in-house ELISA using polyclonal antibodies as catching and tagging antibodies labelled with horseradish peroxidase (DAKO, Glostrup, Denmark). Plasma leptin concentrations were measured with a commercial human leptin RIA kit (Linco Research, St Charles, MO, USA) according to the manufacturer's instructions. The relative plasma content of von Willebrand factor was measured by the Cejka method ${ }^{(37)}$. The inter-assay and intra-assay $\mathrm{CV}$ were 3.3 and $3.6 \%$ (fibrinogen), 6.5 and $3.2 \%$ (CRP), 8.2 and $6.0 \%$ (leptin) and 5.1 and $5.7 \%$ (von Willebrand factor). Total leucocyte counts and relative granulocyte, lymphocyte and monocyte counts were determined on a Coulter Counter (Coulter ${ }^{\circledR}$ GEN.S; Beckmann Coulter Inc., Fullerton, CA, USA) following the manufacturer's instructions.

\section{Covariables}

The following variables were considered potential confounders: socio-economic status (SES) ${ }^{(38)}$, parity (none, one or $\geq$ two children) at study entry ${ }^{(12,39)}$, maternal smoking during pregnancy and anyone smoking at home during the 7-year follow-up period (both measured as total number of cigarettes/1000) ${ }^{(40)}$, breast-feeding (number of months) ${ }^{(41)}$, maternal age at study entry (years) ${ }^{(42)}$, infant $\mathrm{sex}^{(43)}$, gestational age (weeks) ${ }^{(6,12)}$, parent ethnicity (Caucasian or not) ${ }^{(43)}$, birth season (divided into quarters) ${ }^{(44)}$, season of follow-up measurements (divided into quarters) ${ }^{(45)}$, day-care attendance (total number of days/100) ${ }^{(46)}$, parental history of atopy (none, one of the parents, or both) assessed at followup $^{(41)}$ and weight gain during the first year of life corrected for infant sex and age (SD scores) ${ }^{(47)}$. The relative dihomo- $\gamma$ linolenic acid, EPA and DHA concentrations in umbilical cord plasma and vein and artery wall PL, and in maternal plasma PL, as well as the relative AA concentration of plasma PL of the children at follow-up were also selected as potential covariables $^{(48)}$. Exact information on SES was not available. Therefore, parental SES was measured by proxy, using 'income' as an SES indicator, based on the parental postal code at the time of delivery (Geomarktprofiel; Wegener DM, The Netherlands). This information was classified in five groups ranging from 1 (twice or more modal income) to 5 (minimum income); SES values in the categories unknown (0) and diverse (6) were omitted and, thus, reported as missing values. Children who never received breast milk were classified as formula-fed and the remaining children as breast-fed. The following covariables were considered confounders in the models with PEF-related outcomes only: endurance time (time required until maximal exercise was reached, abstracted from the Bruce treadmill test) ${ }^{(28)}$, site of first PEF measurement (at the laboratory with extensive instructions by the research team or at home, with written instructions only) and children's height and weight at follow-up (age 7 years) ${ }^{(49)}$.

\section{Data evaluation and statistical analysis}

All data are presented as medians and interquartile ranges, unless otherwise mentioned. Unadjusted and multivariableadjusted linear and logistic regressions were performed to test the associations between maternal and neonatal AA concentrations and the immune-related variables. Before this, data distributions of the dependent variables were checked by means of the Shapiro-Wilk test; in case of skewness transformation was applied (natural log, square root, square or $1 /$ square) to optimise the data distribution towards normal. When transformation improved but did not normalise the distribution, linear regression analyses were still performed, but results were accepted only if the residuals were normally distributed. If this was not the case, the variables were dichotomised and logistic regression analyses were performed. In the regression analyses with maternal AA levels this appeared the case for absolute monocyte counts and leptin concentrations. Therefore, these variables were analysed as dichotomous variables ( $\geq$ median $v$. $<$ median). The same was done for fibrinogen and leptin concentrations in relation to neonatal AA concentrations. The von Willebrand factor values were biphasically distributed with the point of overlap situated at $68 \%$. Therefore, this variable was analysed as a dichotomous variable also $(\geq 68 v .<68 \%)$. Because the PEF exercise variable had a non-parametric distribution, this variable was also dichotomised. Children without an asthmatic condition usually have a smaller PEF decline $(<15 \%)$ after exercise ${ }^{(23)}$, because they will develop less bronchoconstriction compared with their asthmatic counterparts. Therefore, this value was selected as the threshold for dichotomisation $(\geq 15 v$. $<15 \%)$.

Values of normally distributed variables (either before or after transformation) were considered outliers and removed from the dataset if they were more than four standard deviations away from the mean. Values of not normally distributed variables, 
even after optimal transformation, were considered outliers and were removed from the dataset if their values were more than three interquartile ranges below or above the median.

Insufficient complete cases were available to allow inclusion of all the above-mentioned covariables in the analyses. Therefore, for each of the fifteen dependent variables and each covariable, bivariable regression analyses were performed which included one dependent variable, one explanatory variable and one covariable. These analyses were used to determine which of the covariables contributed to the relationships between dependent and explanatory variables, either as a significant predictor $(P<0.05)$ or as a confounder (if its removal caused the B-value to change at least $10 \%$, and $20 \%$ or more of the standard error of this B-value ${ }^{(50)}$. Only covariables that appeared predictors or confounders were included in the various multivariable-adjusted analyses. For practical reasons, bivariable analyses were only done for AA concentrations in maternal plasma PL at 32 weeks of gestation and for AA concentrations in cord artery wall PL. The selected covariables were subsequently included in all models with corresponding maternal or neonatal AA variables. For all unadjusted and multivariableadjusted regression analyses the same complete dataset was used. Data points suspected of being overly influential were checked by calculation of their Cook's distances and removed if their values were $>1$. Such influential data points were observed in four multivariable-adjusted regression models, but exclusion of these data points did not alter the final results.

Atopy and the three pulmonary function variables were regarded as the dependent variables of primary interest. Relationship studies with these variables were, therefore, considered the primary analyses. All other relationships were regarded of secondary interest. The significance level for the primary analyses was set at a threshold of $P<0 \cdot 05$, with $P<0 \cdot 10$ indicating a non-significant trend. Secondary relationships were considered significant at $P<0 \cdot 01$, to correct for multiple comparisons, and $P<0.05$ indicated a non-significant trend.

SPSS 11.5 for Windows (SPSS, Inc., Chicago, IL, USA) was used for all statistical analyses.

\section{Results}

An overview of the relative concentrations of the maternal and neonatal fatty acids of interest is given in Table 1. The immune-related parameters of the children measured at 7 years of age are presented in Table 2. The maternal and neonatal characteristics screened as covariables are shown in Tables 3 and 4. In Tables 5 and 6, results of the regression analyses are only shown for combinations of dependent and explanatory variables with significant or trend contributions of AA to either the unadjusted or multivariable-adjusted models. Full results are available on request.

\section{Associations between maternal arachidonic acid status and childhood immune-related clinical parameters}

A negative trend was observed for the unadjusted association between the natural log-transformed PEF amplitude and maternal AA levels at the 22nd week of pregnancy $\left(R^{2} 0.016 ; P=0.069\right)$, but this trend was no longer present

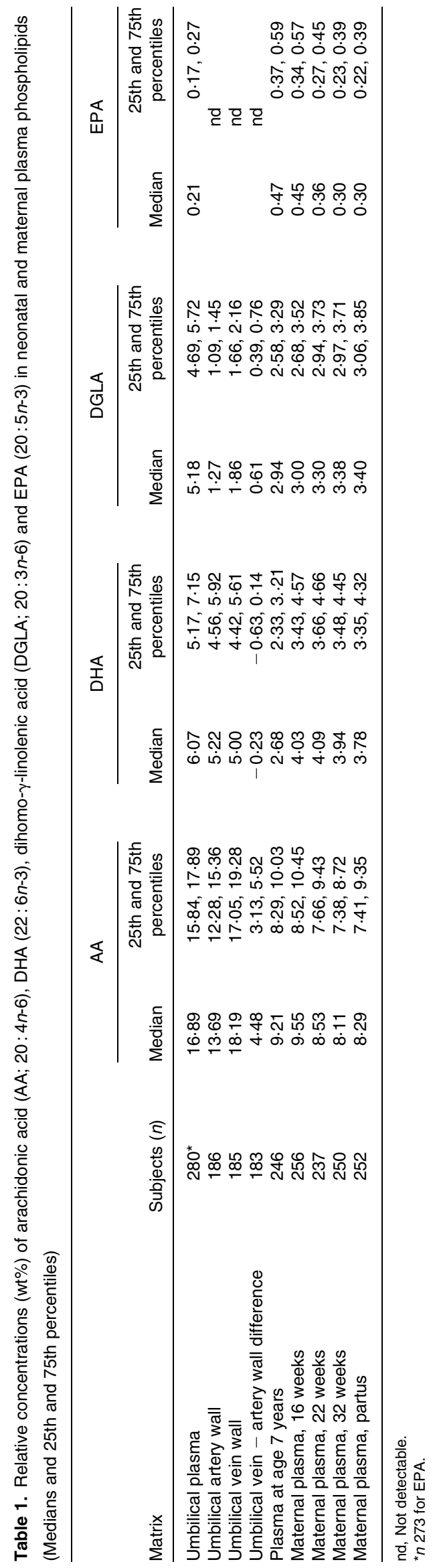


Table 2. Immune-related variables measured in children at follow-up at age 7 years (Medians and 25th and 75th percentiles)

\begin{tabular}{|c|c|c|c|c|}
\hline Variable & $n$ & $\%$ & Median* & 25th and 75 th percentiles \\
\hline \multicolumn{5}{|l|}{ Atopy } \\
\hline No & 183 & 65.4 & & $N / A$ \\
\hline Uncertain & 45 & $16 \cdot 1$ & & $\mathrm{~N} / \mathrm{A}$ \\
\hline Yes & 52 & $18 \cdot 6$ & & $\mathrm{~N} / \mathrm{A}$ \\
\hline PEF morning (litres/min) & 257 & & 214.58 & $193.96,242.50$ \\
\hline PEF amplitude (\%) & 255 & & 7.89 & $5 \cdot 80,10 \cdot 28$ \\
\hline PEF exercise (\%) & 257 & & $5 \cdot 26$ & $0.00,10.53$ \\
\hline Leucocytes $\left(\times 10^{9} / \mathrm{l}\right)$ & 243 & & $6 \cdot 60$ & $5 \cdot 80,7 \cdot 70$ \\
\hline Monocytes ( $\left.\times 10^{9} / \mathrm{I}\right)$ & 243 & & 0.40 & $0.30,0.50$ \\
\hline Granulocytes $\left(\times 10^{9} / l\right)$ & 245 & & 3.60 & $2 \cdot 80,4.60$ \\
\hline Lymphocytes $\left(\times 10^{9} /\right)$ & 246 & & $2 \cdot 50$ & $2 \cdot 10,3 \cdot 00$ \\
\hline Monocytes (\%) & 245 & & $6 \cdot 20$ & $4.65,8.00$ \\
\hline Granulocytes (\%) & 244 & & 53.95 & $47 \cdot 83,62 \cdot 50$ \\
\hline Lymphocytes (\%) & 246 & & 38.95 & $32.00,44.43$ \\
\hline CRP $(\mathrm{mg} / \mathrm{l})$ & 232 & & 0.19 & $0.07,0.58$ \\
\hline Leptin $(\mu \mathrm{g} / \mathrm{l})$ & 245 & & 2.85 & $2.07,3.93$ \\
\hline Fibrinogen ( $\mathrm{g} / \mathrm{l})$ & 233 & & $2 \cdot 60$ & $2.40,2.80$ \\
\hline $\operatorname{vWF}(\%)$ & 233 & & 81.00 & $71.00,84.00$ \\
\hline
\end{tabular}

N/A, not applicable; PEF, peak expiratory flow; PEF morning, average PEF in the morning; PEF amplitude, average PEF daily amplitude; PEF exercise, PEF decline after exercise provocation; CRP, C-reactive protein; vWF, von Willebrand factor.

${ }^{*}$ For all variables non-transformed values are given.

in the multivariable-adjusted analysis (Table 5). A significant positive association and a positive trend were observed for the unadjusted relationships between PEF exercise values and the AA levels in maternal plasma at 16 weeks of pregnancy $(P=0.033)$ and directly after delivery $(P=0.097)$, respectively. In these unadjusted models, differences in AA concentrations explained 4.5 and $2.8 \%$ of the variance in $\mathrm{PEF}$ exercise, respectively. After adjustment of both models for relevant covariables, only a positive trend remained for the first relationship $(P=0.059)$, in which AA explained $3.5 \%$ of the variation in PEF exercise.

For all other combinations of maternal AA concentrations and immune-related clinical parameters no significant associations or trends were found.

Associations between the maternal arachidonic acid status and childhood immune-related plasma variables

The only significant relationship observed in the unadjusted analyses was a positive association between the AA levels at week 32 of pregnancy and fibrinogen concentrations in children's plasma at follow-up (linear regression analysis after quadratic transformation of fibrinogen concentrations; $P=0.006$ ) (Table 5). After adjustment for the child's AA concentration at follow-up, the association lost significance $(P=0 \cdot 014$, which is higher than 0.01 that was set for the secondary analyses) and differences in maternal AA concentrations explained $2.9 \%$ of the variance in fibrinogen levels. Negative trends were observed for the unadjusted associations of the relative and absolute monocyte counts with AA concentrations at the 16th week of pregnancy. The models explained 2.1 and $4.5 \%$ of the variance in relative $(P=0.030)$ and absolute $(P=0.034)$ monocyte counts, respectively. These trends persisted after adjustment for the appropriate covariables, which resulted in models in which the AA concentrations explained $1.8 \%$ (relative monocyte values; $P=0.039$ ) and $4.4 \%$ (absolute monocyte counts; $P=0.030$ ) of the monocyte variance. All other regression analyses revealed no significant associations or trends.

Table 3. Maternal and child characteristics used as continuous covariables

(Medians and 25th and 75th percentiles)

\begin{tabular}{lccc}
\hline Characteristics & $n$ & Median & 25th and 75th percentiles \\
\hline Maternal age at study entry (years) & 280 & 29.5 & $26 \cdot 9,32 \cdot 4$ \\
Gestational age at birth (weeks) & 280 & $40 \cdot 1$ & $39 \cdot 3,41 \cdot 0$ \\
Infant weight gain during first year of life (SD) & 247 & $1 \cdot 0$ & $0 \cdot 3,1 \cdot 7$ \\
Height at 7-year follow-up (cm) & 275 & $127 \cdot 0$ & $123 \cdot 2,130 \cdot 4$ \\
Weight at 7-year follow-up (kg) & 275 & $24 \cdot 5$ & $22 \cdot 2,27 \cdot 6$ \\
Day-care attendance during 7-year follow-up (total days/100) & 272 & 0.08 & $0 \cdot 08,0 \cdot 12$ \\
Breast-feeding (months) & 278 & $0 \cdot 00$ & $0 \cdot 00,3 \cdot 00$ \\
Maternal smoking during pregnancy (total cigarettes/1000) & 280 & 0.00 & $0 \cdot 00,0.54$ \\
Anyone smoking at home during 7-year follow-up (total cigarettes/1000) & 276 & $5 \cdot 29$ & $0 \cdot 00,25 \cdot 6$ \\
Endurance time at 7-year follow-up (min)* & 248 & 10.5 & $10 \cdot 0,11 \cdot 2$ \\
\hline
\end{tabular}

* Time required to reach maximal exercise, calculated from the Bruce test results ${ }^{(28)}$ and considered a measure of physical fitness. 
Table 4. Frequency and code for relevant discrete parental and infant covariables

\begin{tabular}{|c|c|c|}
\hline & $n$ & $\%$ \\
\hline \multicolumn{3}{|c|}{ Parental covariables } \\
\hline \multicolumn{3}{|c|}{ Parents' SES grade (income) } \\
\hline High (1) & 4 & 1.4 \\
\hline Above modal (2) & 23 & $8 \cdot 2$ \\
\hline Modal (3) & 44 & $15 \cdot 7$ \\
\hline Below modal (4) & 74 & $26 \cdot 4$ \\
\hline Low (5) & 31 & $11 \cdot 1$ \\
\hline Missing & 104 & $37 \cdot 1$ \\
\hline Total & 280 & 100 \\
\hline \multicolumn{3}{|c|}{ Parental atopy, positive } \\
\hline None & 148 & $52 \cdot 9$ \\
\hline Father & 44 & $15 \cdot 7$ \\
\hline Mother & 64 & $22 \cdot 9$ \\
\hline Both & 19 & $6 \cdot 8$ \\
\hline Missing & 5 & $1 \cdot 8$ \\
\hline Total & 280 & 100 \\
\hline \multicolumn{3}{|l|}{ Parity } \\
\hline 0 & 193 & 68.9 \\
\hline 1 & 68 & $24 \cdot 3$ \\
\hline$\geq 2$ & 19 & $6 \cdot 8$ \\
\hline Missing & 0 & 0 \\
\hline Total & 280 & 100 \\
\hline \multicolumn{3}{|l|}{ Parents' ethnicity } \\
\hline Caucasian (0) & 270 & $97 \cdot 1$ \\
\hline All other (1) & 8 & $2 \cdot 9$ \\
\hline Missing & 0 & 0 \\
\hline Total & 280 & 100 \\
\hline \multicolumn{3}{|l|}{ Infant covariables } \\
\hline \multicolumn{3}{|l|}{ Infant sex } \\
\hline Male (0) & 152 & $54 \cdot 3$ \\
\hline Female (1) & 128 & $45 \cdot 7$ \\
\hline Missing & 0 & 0 \\
\hline Total & 280 & 100 \\
\hline \multicolumn{3}{|c|}{ Birth season quartiles* } \\
\hline 1 & 71 & $25 \cdot 4$ \\
\hline 2 & 49 & 17.5 \\
\hline 3 & 71 & 25.4 \\
\hline 4 & 88 & 31.4 \\
\hline Missing & 1 & 0.4 \\
\hline Total & 280 & 100 \\
\hline \multicolumn{3}{|c|}{ Season of measurement quartiles* } \\
\hline 1 & 97 & 34.6 \\
\hline 2 & 75 & $26 \cdot 8$ \\
\hline 3 & 61 & $21 \cdot 8$ \\
\hline 4 & 47 & $16 \cdot 8$ \\
\hline Missing & 0 & 0 \\
\hline Total & 280 & 100 \\
\hline \multicolumn{3}{|c|}{ Site of first PEF measurement } \\
\hline At laboratory (0) & 106 & 37.9 \\
\hline At home (1) & 152 & $54 \cdot 3$ \\
\hline Missing & 22 & $7 \cdot 9$ \\
\hline Total & 280 & 100 \\
\hline
\end{tabular}

SES, socio-economic status; PEF, peak expiratory flow.

${ }^{*} 1$ = January-March, 2 = April-June, $3=$ July-September, $4=$ OctoberDecember.

Associations between neonatal arachidonic acid status and childhood immune-related clinical parameters

Unadjusted regression analysis showed a significant negative association between natural log-transformed PEF amplitude values and AA concentrations in neonatal plasma PL $\left(R^{2} 0 \cdot 026 ; P=0 \cdot 020\right)$ (Table 6). However, after adjustment for relevant covariables, this association was reduced and no longer significant. A positive trend was observed for the unadjusted relationship between PEF exercise and the difference in AA concentrations between the walls of cord arteries and cord vein $(P=0.087)$, which explained $4.6 \%$ of the variance in PEF exercise. This positive trend disappeared, however, after adjustment for the appropriate covariables. No significant associations or trends were observed for the other combinations of neonatal AA concentrations and clinical immune-related variables.

\section{Associations between neonatal arachidonic acid status and childhood immune-related plasma variables}

In unadjusted regression analyses, only a positive trend was observed for the relationship between absolute lymphocyte counts (square root-transformed) of the children at follow up, and their plasma PL AA concentrations at birth $\left(R^{2} 0.018 ; P=0.038\right)$, but this trend did not survive after adjustment for dihomo- $\gamma$-linolenic acid and DHA concentrations in neonatal plasma PL (Table 6). In multivariable-adjusted analyses, a negative trend was found for the relationship between the natural log-transformed plasma CRP levels of the children at follow-up and the AA concentrations measured in the PL of their cord artery walls $(P=0.019)$. In this model, AA explained $3.7 \%$ of the CRP variance. A positive trend $(P=0.049)$ was observed for the multivariable-adjusted relationship between children's natural log-transformed absolute leucocyte counts at age 7 years and the AA levels of their cord vein wall PL. In this relationship, AA explained $2.3 \%$ of the variance in leucocyte count. No other important associations or trends were noted (Table 6).

\section{Discussion}

In the unadjusted primary analyses of this prospective mother-child cohort, negative associations were observed between the average PEF daily amplitude (PEF amplitude) of 7-year-old children and the AA concentrations in plasma PL of their mothers at 22 weeks of gestation (reflecting fetal exposure to AA) and in umbilical plasma PL (reflecting prenatal AA exposure). On the other hand, positive associations were observed between the PEF decline after maximal exercise (PEF exercise) and maternal AA concentrations at 16 weeks of pregnancy and directly after delivery, respectively. These positive associations are in concordance with the positive non-significant trend between PEF exercise and neonatal vein-artery AA differences, which we consider a proxy of fetal AA consumption. For these associations and trends, variations in AA levels explained no more than 0.8 to $3.5 \%$ of the variability of these immune-associated variables, strongly suggesting that the prenatal AA status of an individual hardly contributes to these immune-related aspects at 7 years of age. This is also supported by the finding that the associations are not only functionally inconsistent but contrasting as well. Since values for PEF amplitude and/or PEF exercise are usually increased in subjects with an asthmatic condition $^{(23)}$, the negative associations we observed with AA exposure imply a beneficial effect of AA on lung function, if relationships are causal. In contrast, the positive relationships between AA exposure and PEF exercise indicate 
Table 5. Unadjusted and multivariable-adjusted linear (LIN) and logistic (LOG) regression analyses of the relationships between plasma phospholipid (PL) arachidonic acid (AA) concentrations of mothers sampled during pregnancy and at delivery, and immune-related variables measured in their children at 7 years of age

\begin{tabular}{|c|c|c|c|c|c|c|c|c|c|c|c|c|c|c|}
\hline \multirow[b]{2}{*}{ Pregnancy week } & \multirow[b]{2}{*}{$\begin{array}{l}\text { Dependent } \\
\text { variables }\end{array}$} & \multirow[b]{2}{*}{ LIN or LOG } & \multirow[b]{2}{*}{$n$} & \multicolumn{5}{|c|}{ Unadjusted analysis results } & \multicolumn{6}{|c|}{ Multivariable-adjusted analysis results $\dagger$} \\
\hline & & & & $R^{2}$ & B & $\beta$ or OR & $P$ & $95 \% \mathrm{Cl}$ & $R^{2}$ & $\mathrm{~B}$ & $\beta$ or OR & $r^{2}$ & $P$ & $95 \% \mathrm{Cl}$ \\
\hline \multicolumn{15}{|l|}{ Primary analyses } \\
\hline 22 & $\begin{array}{l}\text { PEF amplitude } \\
(\%, \operatorname{In} \ddagger)\end{array}$ & LIN & 207 & 0.016 & -0.04 & -0.13 & 0.069 & $-0.09,0.00$ & 0.040 & $-0.03 \S$ & -0.09 & 0.008 & 0.204 & $-0.08,0.02$ \\
\hline 16 & $\begin{array}{l}\text { PEF exercise } \\
\quad(\geq 15 \% \text { decline })\end{array}$ & LOG & 212 & 0.045 & 0.34 & 1.40 & $0.033^{*}$ & $1.03,1.90$ & 0.077 & $0.33 \|$ & $1 \cdot 38$ & 0.035 & 0.059 & $0.99,1.94$ \\
\hline Delivery & $\begin{array}{l}\text { PEF exercise } \\
\quad(\geq 15 \% \text { decline })\end{array}$ & LOG & 208 & 0.028 & 0.27 & $1 \cdot 31$ & 0.097 & $0.95,1.80$ & 0.050 & $0 \cdot 20 \|$ & $1 \cdot 22$ & 0.014 & 0.251 & $0.87,1.73$ \\
\hline \multicolumn{15}{|c|}{ Secondary analyses } \\
\hline 32 & $\begin{array}{l}\text { Fibrinogen } \\
\quad(\mathrm{g} / \mathrm{l} \text {, squareq) }\end{array}$ & LIN & 208 & 0.036 & 0.28 & 0.19 & $0.006^{*}$ & $0.08,0.47$ & 0.044 & $0.25 \dagger \dagger$ & $0 \cdot 17$ & 0.029 & 0.014 & $0.05,0.45$ \\
\hline 16 & Monocytes (\%) & LIN & 225 & 0.021 & -0.24 & -0.14 & 0.030 & $-0.45,-0.02$ & 0.077 & $-0.22 \ddagger \ddagger$ & -0.13 & 0.018 & 0.039 & $-0.43,-0.01$ \\
\hline 16 & $\begin{array}{l}\text { Monocytes } \\
\quad\left(\geq 0.40 \times 10^{9} / I\right)\end{array}$ & LOG & 139 & 0.045 & -0.27 & 0.77 & 0.034 & $0.60,0.98$ & 0.155 & $-0.30 \S \S$ & 0.74 & 0.044 & 0.030 & $0.56,0.97$ \\
\hline
\end{tabular}

$R^{2}$, coefficient of determination for linear regression, Nagelkerke $R$ square for logistic regression; B, unstandardised regression coefficient; $\beta$, the standardised regression coefficient of $B$ for linear regression (LIN), which is the number of outcome standard deviations that the outcome will change as a result of one predictor standard deviation change in the predictor; OR, OR for logistic regression (LOG); $P, P$ value of $\mathrm{AA}$ contribution; $95 \% \mathrm{Cl}, \mathrm{Cl}$ of $\mathrm{B}$ for linear regression and of OR for logistic regression, both set at $95 \% ; r^{2}$, square of the semi-partial correlation coefficient of AA; PEF, peak expiratory flow.

${ }^{*}$ Significant relationship $(P<0.05$ for primary analyses; $P<0.01$ for explorative analyses). A non-significant trend was indicated as $0.05 \leq P<0.10$ for primary analyses and as $0.01 \leq P<0.05$ for secondary analyses. $\dagger$ The total model $P$ values of the (final) multivariable-adjusted analyses were between 0.000 and 0.178 .

$\ddagger$ Natural log transformation.

$\S$ Covariables: site of first PEF measurement and maternal plasma PL DHA concentrations.

Covariables: DHA concentrations in maternal plasma PL and AA concentration in children's plasma PL at follow-up.

† Covariable: AA concentration in children's plasma PL at follow-up.

$\neq \ddagger$ Covariable: infant sex.

C Covariables: infant sex, DHA concentrations in maternal plasma PL and parents' socio-economic status.

I Square transformation. 
Table 6. Unadjusted and multivariable-adjusted linear (LIN) and logistic (LOG) regression analyses of the relationships between phospholipid (PL) arachidonic acid (AA) concentrations in several umbilical domains of neonates, and their immune-related variables measured at 7 years of age

\begin{tabular}{|c|c|c|c|c|c|c|c|c|c|c|c|c|c|c|}
\hline \multirow[b]{2}{*}{ Umbilical domain } & \multirow[b]{2}{*}{ Dependent variables } & \multirow[b]{2}{*}{ LIN or LOG } & \multirow[b]{2}{*}{$n$} & \multicolumn{5}{|c|}{ Unadjusted analysis results } & \multicolumn{6}{|c|}{ Multivariable-adjusted analysis results $\dagger$} \\
\hline & & & & $R^{2}$ & B & $\beta$ or OR & $P$ & $95 \% \mathrm{Cl}$ & $R^{2}$ & B & $\beta$ or $\mathrm{OR}$ & $r^{2}$ & $P$ & $95 \% \mathrm{Cl}$ \\
\hline \multicolumn{15}{|l|}{ Primary analyses } \\
\hline Plasma & $\begin{array}{l}\text { PEF amplitude } \\
(\%, \text { In } \ddagger)\end{array}$ & LIN & 212 & 0.026 & -0.04 & -0.16 & $0.020^{*}$ & $-0.07,-0.01$ & 0.106 & $-0.03 \S$ & -0.11 & 0.008 & 0.171 & $-0.06,0.01$ \\
\hline $\begin{array}{l}\text { Vein-artery } \\
\text { difference }\end{array}$ & $\begin{array}{l}\text { PEF exercise } \\
\quad(\geq 15 \% \text { decline })\end{array}$ & LOG & 143 & 0.046 & 0.26 & $1 \cdot 30$ & 0.087 & $0.96,1.76$ & 0.130 & $0 \cdot 18 \|$ & $1 \cdot 19$ & 0.010 & 0.396 & $0.79,1.79$ \\
\hline \multicolumn{15}{|c|}{ Secondary analyses } \\
\hline Plasma & $\begin{array}{l}\text { Lymphocytes } \\
\left(\times 10^{9} / \mathrm{l} \text {, square }\right. \\
\text { root } \emptyset)\end{array}$ & LIN & 246 & 0.018 & 0.02 & 0.13 & 0.038 & $0.00,0.03$ & 0.028 & $0.02 \dagger \dagger$ & 0.12 & 0.011 & 0.105 & $-0.00,0.03$ \\
\hline Artery wall & CRP (mg/l, In $\ddagger)$ & LIN & 137 & 0.020 & -0.10 & -0.14 & 0.101 & $-0.21,0.02$ & 0.153 & $-0.19 \neq \ddagger$ & -0.28 & 0.037 & 0.019 & $-0.35,-0.03$ \\
\hline Vein wall & $\begin{array}{l}\text { Leucocytes } \\
\qquad\left(\times 10^{9} / /, \ln \ddagger\right)\end{array}$ & LIN & 162 & 0.023 & 0.02 & $0 \cdot 15$ & 0.054 & $0.00,0.04$ & 0.107 & $0.02 \S \S$ & 0.17 & 0.023 & 0.049 & $0.00,0.04$ \\
\hline
\end{tabular}

$R^{2}$, coefficient of determination for linear regression, Nagelkerke $R$ square for logistic regression; B, unstandardised regression coefficient; $\beta$, the standardised regression coefficient of B for linear regression (LIN), which is the number of outcome standard deviations that the outcome will change as a result of one predictor standard deviation change in the predictor: $\mathrm{OR}, \mathrm{OR}$ for logistic regression (LOG); $P, P$ value of $\mathrm{AA}$ contribution; $95 \% \mathrm{Cl}, \mathrm{Cl}$ of $\mathrm{B}$ for linear regression and of OR for logistic regression, both set at $95 \% ; r^{2}$, square of the semi-partial correlation coefficient of AA; PEF, peak expiratory flow; CRP, C-reactive protein; DGLA, dihomo- $\gamma$-linolenic acid.

* Significant relationship $(P<0.05$ for primary analyses). A non-significant trend was indicated as $0.05 \leq P<0.10$ for primary analyses and as $0.01 \leq P<0.05$ for secondary analyses.

$\dagger$ The total model $P$ values of the (final) multivariable-adjusted analyses were between 0.006 and 0.093

$\ddagger$ Natural log transformation.

Covariables: site of first PEF measurement, maternal age, DGLA and DHA concentrations in neonatal plasma PL, endurance time, parity and infant sex.

Covariables: umbilical vein-artery differences of DGLA and DHA concentrations, first year weight gain, height at follow-up.

I) Square root transformation.

††Covariables: DGLA and DHA concentrations in neonatal plasma PL.

扯Covariables: parity, maternal smoking during pregnancy, anyone smoking at home during follow-up period, first year weight gain, DGLA and DHA concentrations in umbilical artery wall PL and AA concentration in plasma PL of children at follow-up.

$\S \S$ Covariables: birth season, DGLA and DHA concentrations in umbilical vein wall PL and infant sex. 
a potentially adverse effect of AA. Finally, in the multivariable-adjusted analyses most significant results and trends disappeared.

Concerning the secondary analyses with the immune-related plasma markers, one significant positive association was shown between maternal AA concentrations at 32 weeks of pregnancy and plasma fibrinogen concentrations of the children at 7 years of age. For some other associations only trends were revealed but, like the primary analyses, no more than about $4 \%$ of the functional variability was explained by differences in AA concentration. Moreover, observed associations and trends were functionally inconsistent again and therefore a major influence of early AA exposure on these immune-related plasma markers at age 7 years seems unlikely.

Relationships between early-life exposure to $n-3$ and $n-6$ fatty acids and immune-related clinical conditions measured during childhood have been previously studied ${ }^{(51-54)}$. However, as far as we know, only a few studies investigated the associations between prenatal $n-6$ fatty acid exposure and various indicators of immune function in childhood. Newson et al. did not report any significant association between relative AA concentrations in erythrocyte PL of umbilical cord blood and maternal blood collected in a period between 20 weeks of pregnancy and delivery and the prevalence of wheezing or eczema up to 42 months of age after adjustment for confoun$\operatorname{ders}^{(11)}$. Furthermore, Yu et al. also observed no difference in the relative AA levels of umbilical cord blood PL of children who did or did not develop allergic disease during the first 6 years of life ${ }^{(10)}$. Both findings are in line with our observation that plasma AA concentrations before and around birth are not strongly associated with atopic disease in later childhood. Since these previously mentioned studies used shorter periods between fatty acid exposure measurements and assessment of immune-related variables, it can probably be excluded that the interval period of 7 years in the present study was too long to explain the non-significant results.

Galli et al. selected fifty-seven neonates at high risk for developing atopy and reported that all thirteen newborns who developed atopic disease during the first 12 months of follow-up had significantly (20-40\%) lower AA levels in their cord blood PL at birth as compared with their nonatopic counterparts ${ }^{(9)}$. However, no correction was made for potential confounders which may have biased their results. In addition, Sausenthaler et al. suggested that intake of $n-6$ fatty acid-rich foods, such as margarines and vegetable oils, during the last 4 weeks of pregnancy was associated with an increased risk of allergic diseases in children at the age of 2 years $^{(8)}$. Although these foods contain a high content of linoleic acid, the increased risk could also be related to one of the many other components of these products. Furthermore, it is known from a previous study that a higher maternal intake of linoleic acid does not relate to higher AA concentrations in maternal and umbilical plasma $\mathrm{PL}^{(55)}$.

A strong aspect of the present study is that maternal and neonatal fatty acid concentrations were obtained repeatedly during pregnancy and at parturition, respectively. Since maternal and neonatal long-chain PUFA concentrations are strongly correlated ${ }^{(12)}$, this enabled us to investigate the associations between the child's immune-related variables at age 7 years and the AA exposure from the second trimester of pregnancy on.
Although we tested all relevant variables included in the database for their confounding or predicting potentials, residual confounding cannot be excluded, which is a general shortcoming of observational studies. Due to the absence of relevant data, we were unable to correct our analyses for differences in the postnatal AA consumption of the children, which may have contributed to the inter-individual differences in immune outcome variables at age 7 years. However, we did correct for the plasma PL AA concentration at age 7 years, which can be considered a proxy for the postnatal AA intake, since there is a positive relationship between dietary AA intake and plasma PL AA concentration ${ }^{(56,57)}$.

The use of a parent questionnaire to assess the atopic state of the children is clearly inferior to a full clinical evaluation. However, in large-scale observational studies, questionnaires are often the only practical option to gather clinical information. It should be added that the questionnaire that we used was based on the ISAAC questionnaire ${ }^{(21)}$, which has been properly validated ${ }^{(19,20)}$, showing sensitivity and specificity of at least 77 and $81 \%$, respectively. Furthermore, as mentioned before, all questionnaires were 'scored' by an experienced clinician (J. J. E. H.). Therefore, although not optimal, we consider our atopy-assessment method adequate for the present observational study. Finally, we cannot exclude response bias in this cohort study, since informed consent for the 7-year follow-up was obtained for only 300 out of the 750 eligible mother-child pairs.

In the present study, no clear associations were observed between atopy at age 7 years and prenatal AA exposure as reflected by maternal AA concentrations during pregnancy and at delivery and neonatal AA values at birth. In theory, this may result from too-narrow ranges in AA concentrations and/or a too-low atopy incidence in our study population in relation to our sample size. However, the AA concentrations measured in our study population compare very well with maternal $^{(58,59)}$ and neonatal ${ }^{(59,60)}$ values observed in other studies, whereas the atopy incidence in our population is relatively high as compared with the atopy prevalence reported in a worldwide study ${ }^{(61)}$. Since the OR for the atopy risk as a function of the AA concentrations in the various domains are all close to $1(0 \cdot 79-1 \cdot 11$ for the eight domains studied) and their $95 \% \mathrm{CI}$ are rather tight around 1 (the widest interval being $0.66,1.33$ ), we consider the power of the present study sufficient for a reliable conclusion. Comparable conclusions can be drawn for most other immune-associated variables. This supports the absence of relevant associations between the perinatal AA availability and selected aspects of the immune status at the age of 7 years.

In the present study, the same cases were used in the unadjusted as well as the multivariable-adjusted regression models, enabling us to check whether confounding was present and in which direction confounding influenced the model. In general, results were comparable when unadjusted regression analyses were performed with the maximum number of cases available (data not shown).

Finally, in the present study outliers were removed before statistical analyses were applied. Usually an outlier should only be omitted from the statistical analyses if there is a biological explanation as to why this value is inappropriate. Repeating the statistical analyses with the outliers included did not fundamentally alter the results: non-significant results 
did not become significant and vice versa. So, the final results and the conclusions of the present study did not change when the outliers were added in the analyses.

In conclusion, we investigated whether normally occurring differences in prenatal AA availability could be of importance for the later presence of atopy, lung function and plasma inflammation markers. From the results obtained, such an influence seems rather unlikely since physiological differences in prenatal exposure to AA show few, weak and inconsistent associations with differences in several immune-related clinical conditions and plasma markers at 7 years of age. Genetic predisposition and in utero exposure to other factors are possibly more important determinants of these variables than prenatal AA concentrations. However, because of the limitations of the present observational study (mentioned above), the involvement of early AA status in the development of the immunological system cannot be excluded.

\section{Acknowledgements}

The present study was financially supported by DSM Food Specialties (Delft, the Netherlands).

G. H. proposed and supervised the project. P. R. collected most of the data used in the paper. C. E. H. D., G. H and J. G. F. H. designed the statistical plan. J. G. F. H. calculated the PEF values and J. J. E. H. provided the atopy assessment. C. E. H. D. performed the statistical analyses. All authors contributed to the writing of the manuscript.

Moreover, the authors wish to thank Dr C. Thijs for helpful discussions and Dr A. Kester for his statistical advice. We also thank Dr W. J. M. Gerver for his expert advice concerning infant growth and Professor P. C. Calder for valuable comments on the manuscript.

M. B. is employed at DSM Food Specialties and G. H. provided scientific advice to DSM Food Specialties. None of the other authors had any personal or financial conflicts of interest.

\section{References}

1. Calder PC, Krauss-Etschmann S, de Jong EC, et al. (2006) Early nutrition and immunity - progress and perspectives. Br J Nutr 96, 774-790.

2. Calder PC, Yaqoob P, Harvey DJ, et al. (1994) Incorporation of fatty acids by concanavalin A-stimulated lymphocytes and the effect on fatty acid composition and membrane fluidity. Biochem J 300, 509-518.

3. Kew S, Banerjee T, Minihane AM, et al. (2003) Relation between the fatty acid composition of peripheral blood mononuclear cells and measures of immune cell function in healthy, free-living subjects aged 25-72 y. Am J Clin Nutr 77, $1278-1286$.

4. Calder PC (2006) n-3 Polyunsaturated fatty acids, inflammation, and inflammatory diseases. Am J Clin Nutr 83, 1505S-1519S.

5. Tilley SL, Coffman TM \& Koller BH (2001) Mixed messages: modulation of inflammation and immune responses by prostaglandins and thromboxanes. J Clin Invest 108, 15-23.

6. Al MDM, van Houwelingen AC, Kester ADM, et al. (1995) Maternal essential fatty acid patterns during normal pregnancy and their relationship to the neonatal essential fatty acid status. Br J Nutr 74, 55-68.
7. Dunstan JA, Mori TA, Barden A, et al. (2003) Fish oil supplementation in pregnancy modifies neonatal allergen-specific immune responses and clinical outcomes in infants at high risk of atopy: a randomized, controlled trial. J Allergy Clin Immunol 112, 1178-1184.

8. Sausenthaler S, Koletzko S, Schaaf B, et al. (2007) Maternal diet during pregnancy in relation to eczema and allergic sensitization in the offspring at $2 \mathrm{y}$ of age. Am J Clin Nutr 85, 530-537.

9. Galli E, Picardo M, Chini L, et al. (1994) Analysis of polyunsaturated fatty acids in newborn sera: a screening tool for atopic disease? Br J Dermatol 130, 752-756.

10. Yu G, Kjellman NI \& Bjorksten B (1996) Phospholipid fatty acids in cord blood: family history and development of allergy. Acta Paediatr 85, 679-683.

11. Newson RB, Shaheen SO, Henderson AJ, et al. (2004) Umbilical cord and maternal blood red cell fatty acids and early childhood wheezing and eczema. J Allergy Clin Immunol 114, $531-537$.

12. Al MD, van Houwelingen AC \& Hornstra G (2000) Long-chain polyunsaturated fatty acids, pregnancy, and pregnancy outcome. Am J Clin Nutr 71, 285S-291S.

13. Hornstra $G(2000)$ Essential fatty acids in mothers and their neonates. Am J Clin Nutr 71, 1262S-1269S.

14. Rump P, Popp-Snijders C, Heine RJ, et al. (2002) Components of the insulin resistance syndrome in seven-year-old children: relations with birth weight and the polyunsaturated fatty acid content of umbilical cord plasma phospholipids. Diabetologia 45, 349-355.

15. Bakker EC, Ghys AJ, Kester AD, et al. (2003) Long-chain polyunsaturated fatty acids at birth and cognitive function at $7 \mathrm{y}$ of age. Eur J Clin Nutr 57, 89-95.

16. Al MD, van Houwelingen AC, Badart-Smook A, et al. (1995) The essential fatty acid status of mother and child in pregnancy-induced hypertension: a prospective longitudinal study. Am J Obstet Gynecol 172, 1605-1614.

17. Hornstra G, van Houwelingen AC, Simonis M, et al. (1989) Fatty acid composition of umbilical arteries and veins: possible implications for the fetal EFA-status. Lipids 24, 511-517.

18. Thijs C, Houwelingen A, Poorterman I, et al. (2000) Essential fatty acids in breast milk of atopic mothers: comparison with non-atopic mothers, and effect of borage oil supplementation. Eur J Clin Nutr 54, 234-238.

19. Hederos CA, Hasselgren M, Hedlin G, et al. (2007) Comparison of clinically diagnosed asthma with parental assessment of children's asthma in a questionnaire. Pediatr Allergy Immunol 18, $135-141$.

20. Jenkins MA, Clarke JR, Carlin JB, et al. (1996) Validation of questionnaire and bronchial hyperresponsiveness against respiratory physician assessment in the diagnosis of asthma. Int J Epidemiol 25, 609-616.

21. Asher MI, Keil U, Anderson HR, et al. (1995) International Study of Asthma and Allergies in Childhood (ISAAC): rationale and methods. Eur Respir J 8, 483-491.

22. Johansson SG, Bieber T, Dahl R, et al. (2004) Revised nomenclature for allergy for global use: Report of the Nomenclature Review Committee of the World Allergy Organization, October 2003. J Allergy Clin Immunol 113, 832-836.

23. Anonymous (1992) International consensus report on diagnosis and treatment of asthma. National Heart, Lung, and Blood Institute, National Institutes of Health. Bethesda, Maryland 20892. Publication no. 92-3091, March 1992. Eur Respir J 5, 601-641.

24. Reddel HK (2006) Peak flow monitoring in clinical practice and clinical asthma trials. Curr Opin Pulm Med 12, 75-81.

25. Clough JB, Williams JD \& Holgate ST (1992) Effect of atopy on the natural history of symptoms, peak expiratory flow, and bronchial responsiveness in 7- and 8-year-old children with 
cough and wheeze. A 12-month longitudinal study, [published errarum appears in Am Rev Respir Dis 146, 540] Am Rev Respir Dis 143, 755-760.

26. Timonen KL, Nielsen J, Schwartz J, et al. (1997) Chronic respiratory symptoms, skin test results, and lung function as predictors of peak flow variability. Am J Respir Crit Care Med 156, $776-782$.

27. Higgins BG, Britton JR, Chinn S, et al. (1989) The distribution of peak expiratory flow variability in a population sample. $A m$ Rev Respir Dis 140, 1368-1372.

28. Bruce RA, Kusumi F \& Hosmer D (1973) Maximal oxygen intake and nomographic assessment of functional aerobic impairment in cardiovascular disease. Am Heart $J$ 85, 546-562.

29. Rump P, Verstappen F \& Gerver WJ (2002) Body composition and cardiorespiratory fitness indicators in prepubescent boys and girls. Int J Sports Med 23, 50-54.

30. Koh YY, Kang H, Yoo Y, et al. (2005) Peak expiratory flow variability and exercise responsiveness in methacholine-hyperresponsive adolescents with asthma remission. J Asthma 42, $17-23$.

31. Kannel WB (2005) Overview of hemostatic factors involved in atherosclerotic cardiovascular disease. Lipids 40, 1215-1220.

32. Marnell L, Mold C \& Du Clos TW (2005) C-reactive protein: ligands, receptors and role in inflammation. Clin Immunol 117, 104-111.

33. Otero M, Lago R, Gomez R, et al. (2006) Leptin: a metabolic hormone that functions like a proinflammatory adipokine. Drug News Perspect 19, 21-26.

34. Hernandez LR, Lundberg U \& Arocha-Pinango CL (2000) Experimental thrombosis I: relation with fibrinogen and other haemostatic parameters. Thromb Res 99, 295-305.

35. Roitt I, Brostoff J \& Male D (2001) Immunology, 6th ed. Edinburgh: Mosby.

36. Clauss A (1957) Rapid physiological coagulation method in determination of fibrinogen. Acta Haematol 17, 237-246.

37. Cejka J (1984) Performance characteristics of a commercial kit for assay of factor VIII-related antigen. Clin Chem 30, 814-815.

38. Forastiere F, Agabiti N, Corbo GM, et al. (1997) Socioeconomic status, number of siblings, and respiratory infections in early life as determinants of atopy in children. Epidemiology 8, 566-570.

39. Ball TM, Castro-Rodriguez JA, Griffith KA, et al. (2000) Siblings, day-care attendance, and the risk of asthma and wheezing during childhood. $N$ Engl $J$ Med 343, 538-543.

40. Peat JK (1996) Prevention of asthma. Eur Respir J 9, $1545-1555$.

41. Arruda LK, Sole D, Baena-Cagnani CE, et al. (2005) Risk factors for asthma and atopy. Curr Opin Allergy Clin Immunol 5, $153-159$.

42. Infante-Rivard C (1995) Young maternal age: a risk factor for childhood asthma? Epidemiology 6, 178-180.

43. De Swert LF (1999) Risk factors for allergy. Eur J Pediatr 158, 89-94.

44. Nilsson L, Bjorksten B, Hattevig G, et al. (1997) Season of birth as predictor of atopic manifestations. Arch Dis Child 76, $341-344$.
45. Behrendt H \& Becker WM (2001) Localization, release and bioavailability of pollen allergens: the influence of environmental factors. Curr Opin Immunol 13, 709-715.

46. Krämer U, Heinrich J, Wjst M, et al. (1999) Age of entry to day nursery and allergy in later childhood. Lancet 353, 450-454.

47. Singhal A, Cole TJ, Fewtrell M, et al. (2004) Is slower early growth beneficial for long-term cardiovascular health? Circulation 109, 1108-1113.

48. Kelley DS (2001) Modulation of human immune and inflammatory responses by dietary fatty acids. Nutrition 17, 669-673.

49. Morris MG (1999) A novel non-invasive technique for measuring the residual lung volume by nitrogen washout with rapid thoracoabdominal compression in infants. Thorax 54, 874-883.

50. Maldonado G \& Greenland S (1993) Simulation study of confounder-selection strategies. Am J Epidemiol 138, 923-936.

51. Peat JK, Mihrshahi S, Kemp AS, et al. (2004) Three-year outcomes of dietary fatty acid modification and house dust mite reduction in the Childhood Asthma Prevention Study. J Allergy Clin Immunol 114, 807-813.

52. Oddy WH, Pal S, Kusel MM, et al. (2006) Atopy, eczema and breast milk fatty acids in a high-risk cohort of children followed from birth to 5 yr. Pediatr Allergy Immunol 17, 4-10.

53. Marks GB, Mihrshahi S, Kemp AS, et al. (2006) Prevention of asthma during the first 5 years of life: a randomized controlled trial. J Allergy Clin Immunol 118, 53-61.

54. Almqvist C, Garden F, Xuan W, et al. (2007) Omega-3 and omega- 6 fatty acid exposure from early life does not affect atopy and asthma at age 5 years. J Allergy Clin Immunol 119, $1438-1444$.

55. Rump P \& Hornstra G (2002) The $n-3$ and $n-6$ polyunsaturated fatty acid composition of plasma phospholipids in pregnant women and their infants. Relationship with maternal linoleic acid intake. Clin Chem Lab Med 40, 32-39.

56. Nelson GJ, Schmidt PC, Bartolini G, et al. (1997) The effect of dietary arachidonic acid on plasma lipoprotein distributions, apoproteins, blood lipid levels, and tissue fatty acid composition in humans. Lipids 32, 427-433.

57. Thies F, Nebe-von-Caron G, Powell JR, et al. (2001) Dietary supplementation with eicosapentaenoic acid, but not with other long-chain $n-3$ or $n-6$ polyunsaturated fatty acids, decreases natural killer cell activity in healthy subjects aged $>55$ y. Am J Clin Nutr 73, 539-548.

58. van Eijsden M, Hornstra G, van der Wal MF, et al. (2008) Maternal $n-3 n-6$, and trans fatty acid profile early in pregnancy and term birth weight: a prospective cohort study. Am J Clin Nutr 87, 887-895.

59. Grandjean P \& Weihe P (2003) Arachidonic acid status during pregnancy is associated with polychlorinated biphenyl exposure. Am J Clin Nutr 77, 715-719.

60. Dijck-Brouwer DA, Hadders-Algra M, Bouwstra $\mathrm{H}$, et al. (2005) Lower fetal status of docosahexaenoic acid, arachidonic acid and essential fatty acids is associated with less favorable neonatal neurological condition. Prostaglandins Leukot Essent Fatty Acids 72, 21-28.

61. Anonymous (1998) Worldwide variation in prevalence of symptoms of asthma, allergic rhinoconjunctivitis, and atopic eczema: ISAAC. The International Study of Asthma and Allergies in Childhood (ISAAC) Steering Committee. Lancet 351, 1225-1232. 